\title{
Aktivismus und Bildung für nachhaltige Entwicklung in der Schule? Herausforderungen und Chancen im Umgang mit Aktivismus der Schüler/-innen
}

\begin{abstract}
Zusammenfassung
Die Klimaschutz-Bewegung der jungen Menschen, Fridays for Future, auf den Straßen findet Eingang auch im schulischen Raum und wirft Fragen nach Aufgaben und Funktionen formaler Bildung auf. Wie aber können Aktivismus und formale Bildung einhergehen? Welches Potenzial eröffnet sich hierdurch? Welchen Raum bietet Schule und wo gibt es vielleicht auch Grenzen? Der Beitrag zeigt anhand von Erfahrungen und Reflexionen aus der Umsetzung des Projektes Schools for Earth Potenziale, aber auch Herausforderungen für Schulen angesichts eines zunehmenden politischen Engagements von Schüler/-innen auf. Konkrete Handlungsoptionen werden anhand von Aktivitäten der Ernst-Reuter-Gemeinschaftsschule in Karlsruhe diskutiert, die Teil des Projektes ist. Dabei steht im Fokus, inwiefern diese Aktivitäten sowohl den Anforderungen schulischer Bildung als auch den Bedarfen von jungen Aktivist/-innen Rechnung tragen können.
\end{abstract}

Schlüsselworte: Aktivismus, formale Bildung, Partizipation

\begin{abstract}
The climate protection movement of young people, Fridays for Future, on the streets is also finding its way into school and raises questions about tasks and functions of formal education. But how can activism and formal education go hand in hand? What potential does this open up? What space does school offer? And where are limits? Based on experiences and reflections from the implementation of the Schools for Earth project, this article shows potentials, but also challenges for young people's engagement in school. Concrete options for action are discussed on the basis of activities at the Ernst-Reuter-Gemeinschaftsschule in Karlsruhe, which is part of the project. The focus is on the extent to which these activities can take into account both the requirements of school education and the needs of young activists.
\end{abstract}

Keywords: Activism, formal education, participation
Die weltweite Klimabewegung Fridays for Future hat in Deutschland längst auch Schulen erreicht. Schülerinnen und Schüler gehen für den Klimaschutz und die konsequente Senkung von CO2-Emission auf die Straße. Gleichzeitig gehören Schulen nicht nur zu den größten Energieverbrauchern der öffentlichen Hand und bieten entsprechend viel Potenzial, um aktiv für Klimaschutz einzutreten. Sie sind auch die zentralen Lern- und Lebensorte, denen die Aufgabe zukommt, junge Menschen in ihren Kompetenzen zu stärken, die sie befähigen, sich den gesellschaftlichen Herausforderungen einer Welt im Wandel zu stellen. Darüber hinaus ist die Fähigkeit zur gesellschaftlichen und politischen Teilhabe sowie Mitgestaltung der eigenen Zukunft bedeutsam. Hierbei kommt politischer Bildung im Rahmen einer Bildung für nachhaltige Entwicklung, die insbesondere Partizipation und demokratische Beteiligung als Entscheidungsräume auf Augenhöhe fördert, eine wesentliche Rolle zu: In der im Mai 2021 verabschiedeten Berliner Erklärung BNE2030 wird u.a. gefordert, ,jungen Menschen die aktive Mitwirkung an einer nachhaltigen Entwicklung dadurch zu ermöglichen, dass Lernangebote und Möglichkeiten für bürgerschaftliches Engagement geschaffen und sie mit Kompetenzen und Instrumenten ausgestattet werden, um durch Beteiligung an BNE zum individuellen und gesellschaftlichen Wandel beizutragen" (Berliner Erklärung zur Bildung für nachhaltige Entwicklung, 2021). Schule ,steht [...] immer in Beziehung zu gesellschaftlichen Konstellationen, sozialen Transformationen und/oder bildungspolitischen Vorgaben" (Budde 2020, 2017). So sei Politische Bildung als Freiraum zu verstehen, ,in dem alle Menschen durch Bildung politisch handlungsfähig werden können" (Wohnig 2021, S. 43). Damit Menschen politisch handlungsfähig sein können und an gesellschaftlichen Prozessen partizipieren können, sei es notwendig, Erfahrungsräume zu eröffnen und politisches Handeln erfahrbar zu machen (ebd.). Des Weiteren erläutert Wohnig, dass schulische politische Bildung diese beschriebenen (Frei-) Räume nur bedingt eröffnen kann. Umso mehr sei „die Kooperation mit Akteuren der außerschulischen politischen Bildung anzustreben" (Wohnig 2021, S. 44).

So stellt die weltweite Fridays for Future-Bewegung auch in Deutschland Schulen vor besondere Herausforde- 
rungen, die das Spannungsverhältnis von Bildungsauftrag bzw. bildungspolitischen Vorgaben verdeutlichen, folglich zwischen den Vorgaben und dem Engagement: Die Teilnahme der Schüler/-innen an den Fridays for Future Demonstrationen und somit das Fernbleiben des Unterrichts verletzt die allgemeine Schulpflicht. Andererseits machen die jungen Aktivist/-innen von ihrem Recht demokratischen Handelns Gebrauch. Die jungen Aktivist/-innen sehen ihre eigene Zukunft durch die Klimakrise und die gravierenden Auswirkungen, wie etwa die Zunahme der Extremwetterereignisse, in Gefahr: Das Bundesverfassungsgericht in Karlsruhe hat bestätigt, dass die derzeitige Klimapolitik der Bundesrepublik Deutschland nicht ausreicht, die Folgen der Klimakrise zulasten der jüngeren Generationen geht und diese dadurch in ihren Freiheitsrechten verletzt würden.

Die Klimakrise ist eine gesamtgesellschaftliche Herausforderung, zu der sich auch Schule positionieren muss. Hiermit rückt politisches Denken und insbesondere politisches Handeln in den Diskurs schulischer Bildung. Die Förderung politischen Denkens und Handelns in einer Demokratie vor dem Hintergrund gesellschaftlicher Herausforderungen, „heißt demnach erstens, die gesellschaftlichen Verhältnisse zunächst zu analysieren und zu verstehen, sodass der eigene Standpunkt und die Eingebundenheit in die Verhältnisse erkennbar werden. Zweitens muss die Fähigkeit entwickelt werden, sich ein Urteil zu einem politischen Problem oder Konflikt unter Dissensbedingungen in Auseinandersetzung mit Anderen bilden zu können und drittens bedeutet dies, politisch zu handeln, um Einfluss auf das Problem oder den Konflikt zu nehmen" (Wohnig, 2021, S. 48).

Bildung für nachhaltige Entwicklung kann dieses beschriebene Spannungsverhältnis zwischen Schule und Aktivismus - als Form politischer Teilhabe verstanden - über die Förderung der Gestaltungs- und insbesondere der Handlungskompetenzen aufgreifen und gleichzeitig eine pädagogische Antwort bieten, um Räume für kritischen Austausch und Beteiligung in Schule zu schaffen. Schüler/-innen sollen dort unterstützt werden, Verantwortung zu erkennen und zu übernehmen. In allen Schulgesetzen der Bundesländer ist im Bildungsund Erziehungsauftrag der Schule formuliert, Schüler/-innen zu mündigen Bürger/-innen zu bilden, die Grundwerte der Demokratie zu vermitteln und sie zu befähigen, sich für Demokratie einzusetzen und gleichzeitig gesellschaftliche Verantwortung zu übernehmen.

Das Engagement im Rahmen der Fridays for Future-Bewegung und der hieran beteiligten Schüler/-innen verdeutlicht ihr Bewusstsein für die Relevanz politischen Handelns und die Übernahme von Verantwortung für den Klimaschutz. Hieran anknüpfend werden in dem vorliegenden Beitrag entlang der Zusammenarbeit von Greenpeace e.V. und der Ernst-Reuter-Schule in Karlsruhe im Rahmen des bundesweiten Schools for Earth Projekts folgende Fragen aufgegriffen und konkrete Handlungsoptionen aufgezeigt, die sowohl den Anforderungen schulischer Bildung als auch den Bedarfen von jungen Aktivist/-innen Rechnung tragen können: Wie können Aktivismus und formale Bildung einhergehen? Welches Potenzial eröffnet sich hierdurch? Welchen Raum bietet Schule und wo gibt es vielleicht auch Grenzen?

\section{Ernst-Reuter-Schule in Karlsruhe: Struktu- relle Besonderheiten für gelingende BNE an der Schule}

Die Ernst-Reuter-Schule in Karlsruhe (ERS) ist eine Gemeinschaftsschule, die im Schuljahr 2021/2022 320 Kindern Raum zum Lernen, Arbeiten, Entdecken und Leben gibt. Dabei steht das individuelle Potenzial eines jeden Kindes im Vordergrund und soll entsprechend der eigenen Neigungen und Voraussetzungen auf drei Niveaustufen (grundlegendes, mittleres, erweitertes Niveau) gefördert und gefordert werden. Die jeweiligen Lernfortschritte der Kinder werden in Portfolios und Lerntagebüchern dokumentiert und münden schließlich in ausführliche Lernentwicklungsberichte am Ende des Jahres. Durch die Möglichkeit, selbstverantwortlich in variablen Gruppen zu lernen, werden die Schüler/-innen insbesondere im kooperativen und kreativen Arbeiten gefördert, das täglich durch Lehrkräfte, die als beratende und unterstützende Mentor/-innen und Lernbegleiter/-innen fungieren, unterstützt wird. Im Mittelpunkt des Profils und der Philosophie der Ernst-Reuter-Schule steht somit die Erziehung zu selbstbewussten, verantwortungsvollen und fröhlichen jungen Menschen.

Insbesondere drei Säulen des Schul- und Unterrichtslebens zeichnen die ERS in der täglichen Arbeit aus und unterscheidet sie von vielen anderen Schulen im Sekundarbereich: (1) L.E.B.E.N: Um Kindern die Möglichkeit zu eröffnen, zu eben jenen verantwortungsvollen und selbstbewussten Persönlichkeiten heranzuwachsen, braucht es Erfahrungen und zu lösende Herausforderungen im Bereich der sozialen und emotionalen Intelligenzförderung. Sozialkompetenzen müssen trainiert und aufgebaut werden. Dies geschieht am wirksamsten im Erproben dieser Kompetenzen in realen Lebenssituationen, in denen sich eine positive, innere Einstellung und Haltung aufbauen kann. An zwei Schulstunden in der Woche nehmen daher alle Schüler/-innen der ERS am Projektfach L.E.B.E.N. verpflichtend teil. Diese Projekte, wie die Übernahme eines Verantwortungsjobs (z.B. Unterstützungsarbeit in einem Seniorenheim, im Kindergarten oder anderen Einrichtungen), Arbeit an Themen innerhalb der Schulgemeinschaft oder der Gemeinde, können dabei innerhalb der Schule, aber auch außerhalb in der Gemeinde bzw. im Quartier stattfinden. Erst durch die Erfahrung im realen Raum wird die Relevanz bestimmter Themen, wie beispielsweise einer Bildung für Nachhaltige Entwicklung, für die Schüler/-innen sichtbar und erlebbar. Durch aktives Handeln, aber auch Aushandlungsprozesse mit den Akteur/-innen außerhalb der Schule, erwächst so ein klares Handlungsfeld und ein Handlungsauftrag, Veränderungsprozesse selbst anzustoßen und umzusetzen. (2) TheA: Das Lernen der Zukunft muss mehrdimensional angelegt sein, um der Komplexität der Themen und Sachverhalte unserer Gesellschaft gerecht werden zu können. Zusammenhänge ökonomischer, ökologischer, sozialer und technischer Zusammenhänge müssen also in den Vordergrund gerückt werden. Durch TheA (Themenorientiertes Arbeiten) haben die Kinder der ERS die Möglichkeit, zu diesen Themen gemeinsame Ideen und Lösungsstrategien zu entwickeln. Hierfür werden tradierte Fachstrukturen aufgebrochen. An einem Vormittag in der Woche beschäftigen sich die Schüler/-innen mit acht Projekten, die sie mit ihren Gruppen aus nicht mehr als 14 Kindern nacheinander durchlaufen. Die Grundlage für die Konzeption der 
Inhalte von TheA sind dabei einerseits die Inhalte unterschiedlicher Fächer, die so entfrachtet und in die Projektarbeit überführt werden, andererseits die 17 Nachhaltigkeitsziele. Die gemeinsame, gesellschaftliche Verantwortung wird durch TheA in den Mittelpunkt gerückt und gestärkt. Für die Kinder werden Momente der Selbstwirksamkeit eröffnet und die notwedige Übernahme von Verantwortung für ein gemeinsames Anliegen bestärkt (vgl. Pallesche, 2021). (3) Der Rote Salon: Kooperation, cokreatives und partizipatives Arbeiten, gemeinsame Übernahme von Verantwortung und das Schließen von Kompromissen sind wichtige Kompetenzen für das 21. Jahrhundert. Der Rote Salon der ERS ist der Ort, an dem genau diese gemeinsamen Denk- und Entwicklungsprozesse ermöglicht werden sollen. Schüler/-innen, Lehrkräfte, Eltern und Menschen aus dem eigenen Quartier, aber auch neugierige Menschen aus unterschiedlichen Bereichen und Disziplinen kommen im Roten Salon zusammen und überlegen beispielsweise, wie die Schule positiv weiterentwickelt und gestaltet werden soll. Im Vorfeld können dafür Themenvorschläge eingereicht werden, die dann diskutiert werden sollen. Gemeinschaftlichkeit aller Gremien und die Selbstverpflichtung zur Durchsetzung der im Roten Salon ausgehandelten Verabredungen und Ziele bilden dabei die Basis der Kultur des Roten Salons, der in regelmäßigen Abständen als Abendveranstaltung analog oder virtuell durchgeführt wird.

Schule muss in unserer heutigen, dynamisch komplexen Zeit mehr denn je als Ort verstanden werden, an dem Gemeinschaft gelebt und erfahren wird und Demokratieerziehung stattfindet. Wichtigstes Ziel dabei ist es, dass Schüler/-innen sich selbst als Teil eines Veränderungsprozesses wahrnehmen und begreifen, um diesen somit aktiv mitzugestalten.

Auch Greenpeace setzt sich seit Jahren dafür ein, Bildung für nachhaltige Entwicklung fest im Lehrplan zu verankern und setzt dabei auf politische Teilhabe, wissenschaftliche Analyse, Erstellung von Materialien für die Praxis und Projekte. Durch die gemeinsamen Projekte mit Greenpeace öffnet sich die Ernst-Reuter-Schule nach außen, ermöglicht den Schüler/-innen so Momente der Partizipation, der Erfahrung, dass ihre Stimmen gehört werden und, dass sie die Möglichkeit haben, auch außerhalb des Unterrichtes und der Schulmauern gestaltend Einfluss zu nehmen.

\section{Das Projekt Schools for Earth: demokratisch auf dem Weg zur Klimaneutralität}

Das Projekt Schools for Earth von Greenpeace ist ein bundesweites Schulprojekt, das mit den teilnehmenden Schulen einen konkreten Beitrag zur Erreichung der globalen Ziele für nachhaltige Entwicklung und hierbei insbesondere zum Klimaschutz leistet. Mit dem Projekt unterstützt Greenpeace Schulen dabei, die Energie und das Anliegen der jungen Aktivist/-innen, wie beispielsweise Fridays for Future im Kontext Klimaschutz, in Schule aufzugreifen und sich gemeinsam in und aus Schule heraus für den Klimaschutz einzusetzen. Greenpeace hat dafür Instrumente für Lehrkräfte und Schüler/-innen entwickelt, die einem ganzheitlichen Schulentwicklungsansatzes folgen, dem sogenannten Whole School Approach mit dem Ziel, Klimaschutz und Nachhaltigkeit in Schule zu verankern. Schools for Earth unterstützt Schüler/-innen, sich mit ihren Lehrkräften auf den Weg in Richtung Klimaneutralität $\mathrm{zu}$ machen und so Bildung für nachhaltige Entwicklung zu konkretisieren: im Unterricht, im Miteinander, im Alltag der Lern- und Lebenswelt Schule (vgl. Greenpeace e.V.). So wurde beispielsweise in Zusammenarbeit mit dem Institut für Energie- und Umweltforschung Heidelberg (ifeu) ein $\mathrm{CO}_{2}$-Schulrechner entwickelt, mit dem Schulen ihre individuelle Klimabilanz erstellen können. Mit diesem wissenschaftlich fundierten und einfach zu bedienendem Bilanzierungsinstrument können Schüler/-innen mit ihrer Schulgemeinschaft die $\mathrm{CO}_{2}$-Bilanzierung der eigenen Schule ermitteln. Gleichzeitig bietet eine Handreichung konkrete Handlungsimpulse für die Emissionsreduzierung in der eigenen Schule. Schüler/-innen können dann auf einem Klimacheck-Rundgang Klima-Hotspots der eigenen Schule ausmachen. Die Schüler/-innen erkennen so nicht nur konkrete Handlungsspielräume in der Schule, die sie mit ihrer Schulgemeinschaft selbst gestalten können. Sie erfahren auch die Grenzen ihres Handlungsspielraums oder von Verantwortlichkeiten, z.B. bei der Auswahl des Stromanbieters oder Beschaffungsentscheidungen. Insgesamt sollen sich die Schüler/-innen als wichtigen Teil einer handelnden Gemeinschaft erleben und in Diskussionen und Gesprächen erfahren, dass ihre Stimme bedeutsam ist. Selbstwirksamkeit steht damit im Zentrum dieses Schulentwicklungsansatzes.

Neben dem $\mathrm{CO}_{2}$-Schulrechner bietet das Projekt weitere Angebote für Schulen mit dem Ziel, dass Schüler/-innen Wissen erwerben, die Herausforderungen erkennen, in Handeln umsetzen und damit Selbstwirksamkeit erfahren (weitere Informationen unter https://www.greenpeace.de/schoolsforearth)

\section{Die ERS und das Projekt Schools for Earth}

Das Schools for Earth Projekt an der ERS eröffnet für die Schüler/-innen ein Lernen an zukunftsorientierten und gesellschaftlich relevanten Themen. Dieses Lernen ist geprägt von Gemeinschaftlichkeit, Selbstwirksamkeit, demokratischer Mitbestimmung und vor allem von der Übernahme von Verantwortung durch das Treffen von Entscheidungen, insbesondere unter den Bedingungen einer Kultur der Digitalität ${ }^{1}$. Schule ist hierbei zwar weiterhin ein wichtiger Lernort, aber eben nicht mehr der einzige. Das bedeutet, dass sich Schule nach außen öffnen muss und dadurch ermöglicht, dass auch Menschen von außen in die Schule hereingeholt werden, um von deren Expertisen und Erfahrungen im Lernprozess zu profitieren. Man lernt somit von- und miteinander (vgl. Pallesche, 2021). Schools for Earth ist dabei ein wirksames Beispiel für eine Öffnung von Schule nach außen im Sinne von gesellschaftlich notwendigen Gemeinschaftsprozessen als Antwort auf die Herausforderungen der mehrperspektivischen und komplexen Herausforderungen der Zukunft ${ }^{2}$. Mit Schools for Earth wird Bildung für nachhaltige Entwicklung ein zentraler Teil des Schulcurriculums der Ernst-Reuter-Schule und somit auch Teil von veränderten Lehr- und Lernprozessen in der ERS. Gleichzeitig deckt Schools for Earth die Inhalte des Bildungsplans in Baden-Württemberg ab, bei dem Bildung für Nachhaltige Entwicklung eines der sechs Leitprinzipien ist. BNE wird dabei als Querschnittsaufgabe verstanden und betrifft sämtliche Bereiche der Schulentwicklung, auch im Kontext 
einer Kultur der Digitalität, die mit Blick auf BNE untrennbar mitgedacht werden muss (Pallesche, 2021). Dabei ist das Lösen komplexer Problemstellungen und Herausforderungen, in gemeinschaftlichen, cokreativen Prozessen, wie es beispielsweise der Whole School Approach Ansatz von Schools for Earth vorsieht, ein zentrales Merkmal der Kultur der Digitalität.

Dazu bedarf es eines Verständnisses, Zukunft neu zu denken und vor allem kollektiv zu gestalten, was wiederum einen Kulturbegriff voraussetzt, der Kultur als Gestaltungsaufgabe betrachtet (vgl. Krainer \& Trattningg, 2007, S. 10; Pallesche, 2021). Erst durch aktives Tun gelangen Kinder und Jugendliche von der Wissensebene zur Handlungseben, die wiederum zu einer Haltung im Sinne einer Kultur der Nachhaltigkeit (vgl. ebd., S. 11) führt.

Für den Kontext Schule bedeutet diese Erkenntnis, dass Ziele neu definiert werden müssen. Dem Einzelnen fällt es schwer, sich in der Kultur der Digitalität alleine zu orientieren, weswegen gemeinschaftliche Handlungen von zentraler Bedeutung sind, um Handlungsfähigkeit entstehen zu lassen und diese strategisch zu festigen (Stalder 2017, S. 95 u. 129). Dies bedeutet für ein Lernen und Lehren im schulischen Kontext die Notwendigkeit, cokreative und gemeinschaftlich orientierte Lernsettings zu schaffen, die Eigenverantwortung, Kooperations- und Konfliktfähigkeit ebenso stärken und herausbilden wie die Fähigkeit zur Selbstorganisation (vgl. Krainer 2007, S. 348) und natürlich Empathie, Toleranz und Respekt aufzubauen.

Gerade in Bezug auf Bildung für nachhaltige Entwicklung und den komplexen Themenbereich Klima zeigt die Ernst-Reuter-Schule durch die Arbeit und Entwicklung der zuvor skizzierten, handlungsleitenden Schulentwicklungssäulen L.E.B.E.N., TheA und dem Roten Salon, wie eine Kultur der Nachhaltigkeit im schulischen Kontext verankert werden kann und muss, damit auch und gerade bei der heranwachsenden Generation eine noch stärkere Sensibilisierung für das Thema Klima und Nachhaltigkeit erzeugt wird.

Diese kreativen und schülerorientierten Säulen im schulischen Alltag bieten für die Themenbereiche des Schools for Earth Projektes ausreichend Raum und Zeit, diese selbstwirksam und vor allem von den Kindern selbstverantwortlich gestaltet und von den Lernbegleiter/-innen beratend bearbeiten zu lassen. So ist z.B. in TheA das Thema Klimaschutz und Klimagerechtigkeit in den acht Projektbereichen fest verankert. Expertinnen und Experten von Institutionen wie Greenpeace fungieren hierbei speziell als Impuls- und Ratgeber für den Lernprozess der Kinder, wenn sie in die Schule eingeladen werden oder sich mit den Schüler/-innen an außerschulischen Lernorten zusammenfinden.

Über die Öffnung des Unterrichts erschafft die Schule Möglichkeiten, zentral prägende Lern- und Selbstwirksamkeitserfahrungen für die Schüler/-innen zu ermöglichen. In einem interdisziplinär angelegten Projekt im Juli 2021 gelang es beispielsweise erstmals Akteur/-innen aus unterschiedlichen Disziplinen für ein gemeinsames Projekt zum Thema Klima und Nachhaltigkeit zusammenzubringen, mit der Besonderheit, dass die Schülerinnen und Schüler als handelnde Protagonisten im Mittelpunkt standen. Das ZKM Karlsruhe, eines der renommiertesten Museen weltweit, öffnete mit der Ausstellung der „Critical Zones“ seine Türen für die Schüler/-innen der Ernst-Reuter-Schule. Mit dem Greenpeace Bildungsteam, den Ausstellungskurator/-innen und Künstler/-innen, erhielten die Kinder einen vertieften und nachhaltigen Einblick in die Herausforderungen unserer gefährdeten Zukunft und erarbeiteten kreative Lösungsmöglichkeiten - sowohl für die ERS, als auch für das ZKM. Bildung, Klimaschutz und Kunst wurden in diesem Rahmen cokreative und kooperative Gemeinschaftlichkeit miteinander verbunden.

Im Fach L.E.B.E.N. errechneten Schüler/-innen dann gemeinsam mit den Vertreter/-innen von Greenpeace die CO2-Bilanz der Ernst-Reuter-Schule, um daraus Rückschlüsse für notwendig anzupassende Prozesse für den Klimaschutz im eigenen Raum zu definieren. Auch der Rote Salon bot Möglichkeiten, das Projekt weitergehend umzusetzen. Dort diskutieren alle Teilhabenden wiederkehrend und mit Impulsen von Expert/-innen von Greenpeace über Schulentwicklungsthemen im Bereich der Nachhaltigkeit.

Die Ernst-Reuter-Schule in Karlsruhe bietet mit ihren Lehr-Lernformaten die Möglichkeit, gerade diese allumfassenden Themen wie Nachhaltigkeit und Klima in offenen, schülerzentrierten, produktiv- und handlungsorientierten Prozessen verstärkt in den Blick zu nehmen. Gleichzeitig bedarf es aber auch hier großen Mut, Neugier und das klare Selbstverständnis, jeden Schulentwicklungsprozess vom Kind aus zu denken und alle Schüler/-innen partizipativ und bewusst an allen Entwicklungsthemen teilhaben zu lassen. Dies erfordert eine klare Haltung und ein agiles Handeln mit demokratischem Selbstverständnis der Schulleitungen und Unterstützung durch die Schulämter.

\section{Fazit}

Dieses Beispiel zeigt, dass Schule durchaus Angebote machen kann, Aktivismus und Engagement der Schüler/-innen aufzugreifen und diesem Orte der Entfaltung und fachlichen Einbindung zu geben. Hierbei spielt BNE eine wesentliche Rolle, die neben der methodisch-didaktischen Vielfalt, dem fächerübergreifenden Unterricht sowie der inhaltlichen Ausrichtung auf die Stärkung der Zukunftskompetenzen auch Schule als Ganzes in den Blick nimmt und so Schulentwicklungsprozesse bestärken und orientieren kann. Die Arbeit an der ERS zeigt, dass ambitionierte Projekte wie das Schools for Earth-Projekt mit dem ganzheitlichen Schulentwicklungsansatz dann erfolgreich sein können, wenn die bestehende strukturelle Ausrichtung der Schule eben diese Veränderungsprozesse von sich aus ermöglicht. Dies gelingt durch das Zusammenwirken von bereits getroffenen Entscheidungen zur didaktischen Ausrichtung und dazu passenden Projekten: Aktivismus und Engagement der Schüler/-innen, welche oft außerhalb der Schule stattfinden, können in Schule dann Raum finden, wenn die innere Schulstruktur so ausgerichtet ist, dass sie auf äußere Veränderungsprozesse flexibler reagieren kann, beispielsweise durch eine strukturelle Verankerung von TheA. Mit TheA können die Anliegen der Schüler/-innen aufgegriffen und in globale Rahmensetzungen, wie den SDGs, verortet werden. Relevante Kompetenzen werden so, auch durch die Einbeziehung entsprechende fachlicher Expertise, gestärkt. Schulische Bildung an lebens- und alltagsrelevanten Themen kann auf diesem Weg Sinn für die Schüler/-innen 
stiften. Dennoch kommen Grenzen schulischer Bildung darin auf: Im schulischen Kontext finden vor allem formale Lernprozesse statt, die zu einer Qualifizierung oder einem Abschluss führen sollen und einen verpflichtenden Charakter haben. Die Freiheit, die Engagement in seiner Natur der Freiwilligkeit birgt, kann im Kontext der Abschlussprüfungen zu Konflikten und Spannungsfeldern führen. In der Folge bedarf es hier weiterer Abstimmung und Aushandlung mit Akteur/-innen des Schulkontextes, wie beispielsweise den Eltern, die Sicherheit über Abschlüsse und Qualifikationen verlangen.

Die Zusammenarbeit von Greenpeace und der ERS, aber auch mit dem ZKM hat gezeigt, dass die Öffnung der Schule und das Einbeziehen vielfältiger Akteur/-innen Nährboden bieten können, den Lernort Schule zu erweitern und konkretes Schülerhandeln zu begleiten und zu unterstützen. Selbstwirksamkeitserfahrungen der Schüler/-innen können ermöglicht werden, indem eigene Ideen zu Klimaschutzmaßnahmen ernst genommen werden. Schüler/-innen werden dann zu Gestalter/-innen und Expert/-innen, innerhalb und außerhalb der eigenen Schule. „Bildung für nachhaltige Entwicklung [erschöpft] sich also nicht in der Kompetenzentwicklung, sondern ist auch ein Bildungskonzept, das einen kritischen Wertediskurs eröffnet" (Rieckmann 2020, S. 13), insbesondere einen politischen Diskurs durch politische Bildung, um auch dem Aktivismus und dem Engagement der jungen Generation auch im Raum Schule Rechnung zu tragen.

\section{Anmerkungen}

1 Die „Kultur der Digitalität“ ist ein von Stalder (2017) geprägter Begriff. Er impliziert veränderte Lern- und Arbeitsformen in einer sich zunehmend digitalisierten Welt. Dabei beschreibt Stalder, dass Referentialität, Gemeinschaftlichkeit und Algorithmizität zentrale Bestandteile der Kultur der Digitalität sind. So werden in der digitalen Welt beispielsweise Veröffentlichungen zunehmend referenziert, da sie kommentiert, digital bearbeitet oder weiterverarbeitet werden können. Gleichzeitig finden diese Prozesse der Referenzierung in gemeinsamen Aushandlungsprozessen unterschiedlicher Akteuer/-innen statt (Gemeinschaftlichkeit). Aufgrund des hohen Datenaufkommens und dem Wegfallen klassischer "Gatekeeper“, wie beispielsweise Redakteur/-innen, die Inhalte vor der Veröffentlichung sichten und bewerten, übernehmen das in der Kultur der Digitalität zunehmend Algorithmen (vgl. Stalder, 2017).

2 Link zum Projektvideo Schools for Earth an der ERS: https://www.youtube.com/ watch?v=pNyYvZYAoik

\section{Literatur}

Budde, J. (2020). Die Fridays-for-Future-Bewegung als Herausforderung für die Schule. Ein schulkritischer Essay. Die deutsche Schule, 112(2), 216-228. https://doi. org/10.31244/dds.2020.02.08

Greenpeace e.V. (2020). Co2 Schulrechner. https://co2-schulrechner.greenpeace.de/ node/ 1

Greenpeace e.V. (2020). Whole School Approach. Ganzheitlicher Ansatz zur Schulentwicklung. Zugriff am 14.09.2021 https://www.greenpeace.de/sites/www.greenpeace.de/files/publications/sfe_handreichung_wsa_210419.pdf.

Greenpeace e.V. (2021). Unsere Schule für das Klima! Klimaschutzmaßnahmen für Schüler:innen. Zugriff am 14.09.2021 https://www.greenpeace.de/sites/www. green peace.de/files/publications/sfe_handreichung_klimaschutzmassnahmen_210416.pdf

Krainer, L. \& Trattnigg, R. (2007). Kulturelle Nachhaltigkeit. Konzepte, Perspektiven, Positionen. München: Oekom.

Ommert, A. (Hrsg.) (2016). Ladyfest-Aktivismus. Queer-feministische Kämpfe um Freiräume und Kategorien. Queer Studies, Bd. 14. Bielefeld: Transcript. https://doi. org/10.14361/9783839436509

Pallesche, M. (2021). Kulturelle Nachhaltigkeit als Leitperspektive an Gemeinschaftsschulen. In. C. Sippl \& E. Rauscher (Hrsg.). Kulturelle Nachhaltigkeit lernen und lehren Innsbruck. Wien: Studienverlag.

Rieckmann, M. (2020). Bildung für nachhaltige Entwicklung. Von Projekten zum Whole-Institution Approach. In S. Kapelari (Hrsg.). Vierte „Tagung der Fachdidak tik" 2019. Interdisziplinäre fachdidaktische Diskurse zur Bildung für nachhaltige Entwicklung (11-44). Innsbruck: innsbruck university press. https://doi. org/10.15203/99106-019-2-03

Stalder, F. (2017): Kultur der Digitalität. Berlin: Suhrkamp.

UNESCO/BMBF (2021). Weltkonferenz zu Bildung für nachhaltige Entwicklung. Berliner Erklärung zur Bildung für nachhaltige Entwicklung. Zugriff am 14.9.2021 https://en.unesco.org/sites/default/files/esdfor2030-berlin-declaration-ger.pdf

Wohnig, A. (2021): Gesellschaftliche Herausforderungen gemeinsam bearbeiten! Potentiale schulisch-außerschulischer Bildungskooperationen für die Förderung politischen Handelns. Pädagogische Horizonte, 5(1), S. 43-59

\section{Katarina Roncevic}

arbeitet im Bildungsteam bei Greenpeace e.V. und ist Doktorandin an der Universität Vechta. Ihre Arbeits- und Forschungsschwerpunkte sind BNE, inklusive Bildung und Whole School Approach. Sie ist Mitglied des internationalen ESD Expert-Net.

\section{Micha Pallesche}

ist Schulleiter an der Ernst-Reuter-Schule in Karlsruhe. Er ist Doktorand an der Pädagogischen Hochschule Karlsruhe, Mitglied in mehreren nationalen Gremien zum Thema digitale Bildung. Seine Forschungsschwerpunkte sind die Kultur der Digitalität im Kontext von BNE sowie schulische Transformationsprozesse. 